\title{
¿COVID-19 la pandemia y que sabemos de ella? Y como la afronta el Perú.
}

COVID-19 the pandemic and what do we know about it? And how Peru faces it.

Karin J. Vera-López, Badhin Gómez, Rita Nieto-Montesinos, Jorge Alberto Aguilar-Pineda, Miguel Angel Chavez Fumagalli, Diego E. Valencia, Gonzalo Dávila-del-Carpio, Christian L. Lino- Cárdenas. Universidad Católica de Santa María. Arequipa Perú.

\section{INFORMACIÓN}

\section{Historia del Artículo}

Recepción: 26/04/2020

Revisión: 25/05/2020

Aceptación: 26/05/2020

\section{Palabras Clave}

COVID-19,

pandemia, Perú.

\section{Key Words}

COVID-19,

pandemic, Perú.

SARS-CoV-2,

\section{DOI}

https://doi.org/10.35286/veritas. v21i 2.284

\section{RESUMEN}

La enfermedad del coronavirus o COVID-19, fue reportada por la primera vez en diciembre del 2019, en la ciudad de Wuhan China. A la fecha más de 6777435 casos de COVID-19 y 395 597 muertes han sido reportadas globalmente. El brote del COVID-19 es causado por el virus SARS-CoV-2, siendo el brote más grande de las últimas décadas. Por lo cual la Organización Mundial de Salud (OMS) declaró al COVID-19 como pandemia (marzo 11, 2020), asociada con una importante morbilidad y mortalidad. Esta síntesis bibliográfica recapitula las evidencias científicas y los reportes publicados en revistas médicas. El contenido de este manuscrito recoge las opiniones y experiencias de especialistas. Y presentamos nuestra interpretación y recomendaciones a gobierno peruano.

\begin{abstract}
The novel coronavirus disease 2019 or COVID-19, had been reported for the first time in December 2019, in Wuhan China. To date, around 6777435 cases of COVID-19 were confirmed and 395597 deaths worldwide. The COVID-19 outbreak is the largest in the past decades and is caused by a newly discovered coronavirus (SARS-CoV-2). On March 11th, 2020, the world Health Organization (WHO) has declared the COVID-19 a "Pandemic" with high rate of morbidity and mortality. This review synthesizes the scientific evidence and reports in the literature. The content of this manuscript presents the opinions of specialists and we present our interpretation and recommendations to the Peruvian government.
\end{abstract}

\section{EI virus}

El COVID-19 fue nombrado por la OMS y denominado por el Comité Internacional de Taxonomía de Virus como SARS-CoV-2. Este virus tiene una similitud genética con 2 derivados encontrados en los murciélagos. El virus utiliza proteínas glicosiladas en forma de espiga de corona para entrar a las células humanas donde se une al receptor de la enzima convertidora de angiotensina 2 (ECA2) (1) (Figura 1). En humanos, la enzima ECA2 es mayormente expresada en las células alveolares de tipo II (células residentes del pulmón). Sin embargo, los anticuerpos monoclonales que son usados para inhibir el SARS-CoV-1, no han mostrado interacción con el SARS-Cov-2, confirmando la existencia de un nuevo virus. Un estudio reciente ha demostrado que el SARS-CoV-2 no es un producto de laboratorio, pero si un producto de la selección natural de transmisión del animal al hombre. Esta adaptación consta en la adquisición de grupos de glicanos que le confieren selectividad al receptor humano ECA2 (2). Otra vía por la cual el virus ingresa a las células humanas podría ser a través de la proteasa de serinas la TMPRSS2 (1), la cual activa la subunidad S2 de la

Correspondencia:

Karin J. Vera-López

kvera@ucsm.edu.pe glicoproteína en forma de espiga y así facilitar la fusión del virus con la membrana celular (1).

\section{Epidemiologia}

Actualmente el rango de incubación del virus varia de 1 a 14 días con una media de 5 a 6 días. Sin embargo, algunos casos han reportado periodos de incubación de 24 días (3). El virus principalmente se transmite de persona a persona a través de gotas respiratorias, las cuales se liberan cuando la persona infectada tose, estornuda o habla; adicionalmente se ha encontrado en desechos fecales y sangre. El contagio también puede suceder si se tiene contacto con una superficie infectada, y luego hay contacto con zonas sensibles como ojos, nariz o boca.

Datos recientes sugieren que SARS-CoV-2 puede permanecer más tiempo en heces de niños ( 1 a 2 semanas) que en las de adultos. Lo cual sugiere que otros modos de transmisión pueden ser factibles (4). Si se confirma que el virus se puede multiplicar en las heces, originaria un cambio dramático de los protocolos de precaución y aislamiento. Al igual que la transmisión de otros coronavirus, éste es asociado a la infección en centros médicos como el mayor medio de transmisión (40 a 50\%). Estudios en ambientes hospitalarios han demostrado que el virus puede permanecer en los servicios higiénicos, así como los lavados, siendo el 
plástico el material que alberga al virus por más tiempo (60 horas) y seguido por el metal (20 horas) (5).

Estudios adicionales sugieren que el SARS-CoV-2 podría permanecer suspendido en el aire por 1 o 2 horas dependiendo de factores como el calor y la humedad. Al evaluar habitaciones de los pacientes con COVID-19 se ha encontrado ARN viral en muestras de aire y sus sistemas de ventilación $(6,7)$. Los científicos han demostrado que SARSCoV-2 también puede durar hasta tres días en plástico y acero inoxidable, 4 horas en cobre y 24 horas en cartón (8). Estos últimos estudios justificarían las medidas tomadas por los gobiernos de diferentes países incluyendo el peruano donde se recomienda el aislamiento social, el uso de mascarillas y el lavado de manos frecuente.

Se estima que el número de transmisiones causadas por un infectado (R0) oscila entre 2 a 3 personas (Figura 2). Aún más porque la transmisión a partir de personas con SARSCoV-2 y asintomáticas puede ser posible, lo cual tendría un impacto en los esfuerzos de prevención (diagnostico) y aislamiento de pacientes. Además, se ha visto que gran parte de las infecciones secundarias ha sido por contactos en el hogar, de personas que asistieron a reuniones masivas, por falta de equipos de protección y por haber permanecido en entornos aislados y cerrados como los cruceros $(9,10)$. La infección congénita poco probable, aunque se ha informado de una posible transmisión vertical; así mismo los recién nacidos no han presentado complicaciones ni problemas en el parto (11). Aún no existe suficiente información para la evaluación de secuelas de madres infectas en el primer o segundo trimestre.

A pesar de estas observaciones y múltiples reportes científicos, es difícil utilizar los datos existentes para predecir el comportamiento del virus en regiones del mundo tan diferentes como el Perú. Se pensó que la curva exponencial del brote en China se debía a un factor climatológico. Dado que en esa región del globo el invierno se estaba iniciando. Sin embargo, esta idea fue rápidamente contrapuesta al ver que dicha curva de brote fue parecida en países como Italia, España e Irán donde la temperatura es considerablemente cálida. En Estados Unidos la curva del brote se encuentra en pleno crecimiento cuando el invierno está terminando. Esto sugiere que la trasmisión del virus no sigue una dinámica y que el virus se puede adaptar muy rápidamente. Sin embargo, cuando miramos a países como Hong Kong, Corea, Japón y Alemania donde el brote ha podido ser controlado dado a sus rápidas acciones para diagnosticar e informar a la población se pone en evidencia que el diagnóstico rápido es crítico para poder controlar el crecimiento exponencial del brote.

A la fecha (junio 15, 2020), el Perú ha reportado 229736 casos confirmados, de los cuales el $58 \%$ se encuentra en Lima. Utilizando los datos de tasa de contagio en el Perú (Tabla 1), se ha realizado el análisis de datos (Figure 3 ) y su ajuste a un tipo de curva logística es muy relevante, debido a que su R2 presenta un valor de 0.99999 y el valor de X2 de 0.53858 indica una elevada correlación de los datos a este tipo de ajuste. Desde el modelo matemático logístico, podemos sacar la tasa de incremento en el número de pacientes infectados, ambos modelos matemáticos se presentan en la fórmula 1.

Con los datos del ajuste logístico hemos realizado proyecciones de la curva de transmisión a 30, 60, 90 y 120 días, para de esta forma lograr estimar cuando se llegará a la región asintótica de la ecuación matemática, la cual nos habla del control de la epidemia o en términos epidemiológicos, a la culminación de nuevos casos de pacientes infectados. En la figura 4 (A), se presenta la proyección de los infectados como la tasa de incremento a los treinta días de iniciado el brote infeccioso en nuestro país, no pudiéndose visualizar aun alguna disminución en el número de infectados, al contrario, un posible incremento de estos ya que la velocidad de contagio aun presenta una pendiente positiva. En la figura 4 (B), de nuestra estimación predictiva se desprende que aproximadamente a los 48 días del proceso infeccioso se iniciara una disminución de los infectados por que la curva de la tasa de incremento cambia en el valor de su pendiente de positiva a negativa, aunque eso no signifique que el número de infectados detectados se detenga. En la figura $4(\mathrm{C})$, donde mostramos nuestras estimaciones predictivas a los 90 días del proceso infeccioso, podemos observar que se presentara una disminución sostenible del número de infectados detectados y la curva de la tasa de contagio se hallara en un franco descenso hacia su región de decaimiento gaussiano asimétrico, esto se acentúa completamente en la figura 4 (D) (predicción a los 120 días) e ingresando a la región asintótica, lo cual estaríamos controlando el proceso infeccioso en nuestro país.

El fenómeno de nuestra curva respecto a los datos que podemos observar a los dieciocho días, en el cual se pudo espera que se vislumbrase la anhelada región asintótica, fue frustrada por el relajamiento del aislamiento social, y por los constantes desacatos de la población a las medidas de cuarentena dictadas por el estado. Por lo que si deseamos que este escenario realmente desalentados para la población y la economía de nuestro país, donde el $80 \%$ de los empleos de índole independiente, y que podría afectar terriblemente a la ya alicaída economía nacional, creemos que las medidas de aislamiento deben en estas próximas semanas acentuarse completamente, incluso con un toque de queda completo, de las 24 horas, permitiéndose solamente salir a abastecer a los hogares a un miembro de la familia adecuadamente identificado, esta medida drástica afectara en el modelo en forma drástica los parámetros A2 y x0, que nos hablan del número final de infectados y del valor medio diario de infectados por día.

\section{Características clínicas}

Estudios provenientes de los hospitales en Wuhan, China, sugieren que la edad media de los pacientes es 50 años, con una leve inclinación o predomino masculino (12). En varias cohortes de pacientes hospitalizados se ha visto un rango de edad de 49 a 56 años $(9,13)$. Asimismo, tales estudios revelaron que aproximadamente el $25 \%$ de pacientes son casos severos requiriendo cuidados intensivos y aproximadamente el $10 \%$ requiere ventilación mecánica $(14,15)$. En general entre el $83 \%$ y $98 \%$ de pacientes presentan fiebre, $76 \%$ a $82 \%$ presenta fatiga y del $11 \%$ al $44 \%$ presentan mialgias (16), como se aprecia en la Tabla 2.

Entre las observaciones anormales de laboratorio se incluye linfopenia (70\%) (17), tiempo de protrombina prolongado (58\%) (18) y concentraciones elevadas de lactato deshidrogenasa (40\%) (19). Las radiografías de tórax son caracterizadas por infiltrados bilaterales y las tomografías computarizadas se caracterizan por infiltrados en forma de polvo de vidrio $(19,20)$. Estudios histológicos en tejido post- 
mortem indican formación de membrana hialina, infiltrados intersticiales mononucleares inflamatorios y células gigante multinucleares $(21,22)$.

Una revisión reciente de 72314 casos realizada por el Centro Chino para el Control y la Prevención de Enfermedades mostró que menos del $1 \%$ de los casos fueron en niños menores de 10 años (4). A diferencia de los adultos infectados, la mayoría de los niños infectados parecen tener un curso clínico más leve (23). Las infecciones asintomáticas son frecuentes (24). La determinación del potencial de transmisión de estos pacientes asintomáticos es importante para guiar el desarrollo de medidas para controlar la pandemia en curso.

Últimos reportes han señalado que algunos pacientes con la COVID-19 han sufrido episodios de anosmia (perdida del olfato) y disgeusia (perdida del gusto). Esto ha llevado a realizar observaciones más profundas en pacientes con la enfermedad y se ha concluido que la perdida de estos sentidos son una clara señal de la presencia de infección por el virus SARS-CoV-2. Por lo cual se recomienda a las personas que experimenten perdida de olfato o del gusto, aislarse y contactar a personal médico adecuado para el descarte de la enfermedad (25).

\section{Casos versus fatalidad}

Como las manifestaciones clínicas, la tasa de mortalidad (TM) parece ser muy variable (26). Algunos reportes sugieren que entre el $8 \%$ y $15 \%$ de pacientes en edad avanzada fallecen a causa de síndrome respiratorio agudo causado por la COVID-19 (27). La tasa de mortalidad incrementa en los adultos mayores con condiciones mórbidas (28). Sin embargo, hay reportes que indican una mortalidad entre $1 \%$ y $2 \%$ pero eso depende de la eficacia en el diagnóstico y detección de aquellos infectados con síntomas moderados (29). La comparación entre la TM en China e Italia son idénticas en 2.3 (26). Además, las muertes son similares en ambos países con muertes en la mayoría de los ancianos con comorbilidades conocidas (30).

\section{Diagnóstico y pruebas de laboratorio}

La OMS recomienda realizar el diagnostico basado en el historial de viajes (especialmente aquellos que visitaron la provincia de Hubei en China), o en el contacto con personas diagnosticadas o con diagnostico pendiente para el SARSCoV-2 y que presenten fiebre o síntomas en los últimos 14 días (31). Sin embargo, el número creciente de casos sin historia en países como Corea del Sur (32), Italia (29), España (33) y Japón cambiará las recomendaciones de diagnóstico.

A la fecha, la capacidad de analizar las muestras de pacientes es muy limitada. El Centro de Control y Prevención de Enfermedades de los Estados Unidos (CDC), ha publicado las secuencias de cebadores (iniciadores o primers) y protocolos para facilitar la creación de kits de laboratorio para el diagnóstico (34). En contraparte, algunos investigadores han sugerido que dichos cebadores podrían estar causando errores en diagnóstico. Por lo cual se ha pedido a los laboratorios de diferentes universidades y hospitales de apoyar con el diagnóstico molecular por el método de la reacción en cadena de la polimerasa con transcriptasa inversa en tiempo real (RT-PCR), usando muestras nasofaríngeas y raspado bucal o esputo $(35,36)$. El cultivo viral no está recomendado. Análisis adicionales como el recuento de células sanguíneas y análisis microbiológico son también recomendados. Nuevos reportes han sugerido que el virus se puede encontrar en los desechos fecales de pacientes con la COVID-19, por periodos que van de 3 a 7 días (34).

En consecuencia, el diagnóstico molecular por RT-PCR es el método más usado y confiable hasta la fecha. La guía de diagnóstico en el sistema sanitario americano incluye el doble descarte de la prueba de laboratorio, siendo la segunda prueba 48 horas después de la confirmación del caso. Así mismo, pacientes considerados como recuperados requieren pasar una segunda prueba 72 horas después del alta médica (34, 37, 38).

En el Perú las pruebas de laboratorio están centralizadas en el Instituto Nacional de Salud (INS) en Lima, haciendo que el descarte de nuevos casos en provincias demore entre 5 a 8 días. Esto sugiere que, en caso de brote de la epidemia, el diagnostico de pacientes en provincias sería 10 veces más lento que la velocidad de transmisión la cual se calcula en un incremento de 6 veces por día. Estos hechos han motivado la creación de laboratorios de diagnóstico molecular en regiones como Arequipa, Cusco, Tumbes y La Libertad.

Si bien la descentralización va a permitir un diagnóstico molecular rápido de nuevos casos de COVID-19, los nuevos laboratorios a cargo deben cumplir estrictamente los estándares de bioseguridad como lo sugiere la OMS. Así, la guía provisional emitida por dicha entidad el 12 de febrero del presente, recomienda que todo diagnóstico y análisis por PCR de muestras clínicas tomadas de pacientes sospechosos o confirmados, se realicen de acuerdo con las prácticas y procedimientos descritos para laboratorio con nivel de bioseguridad $2+($ BSL2 + ). Más importante aún, el manejo de material con altas concentraciones de virus vivos (ensayos de propagación, aislamiento o neutralización) o grandes volúmenes de materiales infecciosos debe ser realizado solo por personal debidamente capacitado y competente en laboratorios que cumplan requisitos $\mathrm{y}$ prácticas de nivel de bioseguridad 3 (BSL-3) (39). De no contar con tales laboratorios en las diferentes regiones del Perú, la implementación y acreditación de éstos debería ser un objetivo primordial tanto en la academia como en el sector salud, lo cual permitirá hacer frente a nuevas pandemias nacionales y mundiales

\section{Prevención, contención y mitigación de la transmisión}

A la fecha no existe vacuna contra el SARS-CoV-32 y más de 11 vacunas candidatas y más de 100 moléculas ya están en desarrollo o en ensayos clínicos $(40,41)$. El centro nacional de salud de Estados Unidos espera producir una vacuna basada en el ARN mensajero (ARNm) para fines de marzo del presente año. Sin embargo, esto no quiere decir que dicha vacuna estará disponible hasta antes de fines del 2020 .

Dado que el contagio en los centros de trabajo es muy probable, se recomienda que los trabajadores utilicen protección incluyendo mascarillas y lentes que cubran los ojos. Los trabajadores del sector médico deben usar batas, guantes de látex y respiradores con filtro $\mathrm{N}^{\circ} 95$ o algún 
equipamiento que permita la purificación del aire que respiran.

Se recomienda que las personas con síntomas o sospechas de contagio no acudan al hospital como primera medida. Siendo el aislamiento la medida más prudente, luego deberán comunicarse con el Ministerio de Salud (MINSA), para programar el traslado adecuado a centro médico. Las recomendaciones para hospitales en caso de brote son:

- Crear unidades de cuidados intensivos exclusivos para pacientes con COIVD-19.

- Organizar un área de triaje donde los pacientes críticos puedan recibir ventilación mientras se espera los resultados de diagnóstico molecular.

- Establecer protocolos de triaje para pacientes con síntomas, para que sean evaluados rápidamente $\mathrm{y}$ dependiendo del diagnóstico molecular, sean reubicados en la unidad apropiada.

- Asegurarse que el personal médico cuente con equipamiento y protección adecuado. Sobre todo, entrenar y capacitar al personal médico y otros que estén con riesgo alto de contagio.

- Reportar cada caso positivo o sospecha de paciente crítico con la enfermedad COVID-19 al centro regional de coordinación.

Las precauciones a tomar para contención de la transmisión con respecto a los ambientes de aislamiento, consideran que los pacientes sean separados dependiendo del grado de protección del personal médico.

Para el manejo de pacientes asintomáticos y sintomáticos moderados se recomienda quedarse en casa hasta que los síntomas mejoren. El diagnóstico molecular es necesario, pero no mandatorio. Para los pacientes sintomáticos moderados con signos de empeoramiento, se recomienda cuidado médico ambulatorio, vía telefónica o video conferencia. Para los pacientes sintomáticos se recomienda evaluación médica, incluyendo visita médica, diagnóstico molecular y seguimiento vía telefónica. El diagnóstico molecular para el SARS-CoV-2 debe estar disponible para pacientes con síntomas significativos y extender el estudio a las personas en contacto con el paciente. Es claro que el personal médico que fue confirmado positivo para el COVID-19, deberá permanecer en casa hasta que los síntomas desaparezcan. En casos que el flujo de pacientes sobrepase los límites de los hospitales, se debe iniciar la instalación de hospitales móviles y reforzar las guías de procedimiento en casos de emergencias.

\section{Terapia y cuidado clínico}

El cuidado y tratamiento de un paciente con COVID-19 es similar a aquel dado a cualquier paciente con neumonía. Principalmente consta del tratamiento de los síntomas y ventilación asistida, si fuese necesario. Los corticoesteroiedes como la METILPREDNISILONA(42) no son recomendados. El REMDESIVIR (43) en combinación con el interferón beta parecen tener un efecto terapéutico en los pacientes críticos de China y Estados Unidos. El REMDESIVIR es un profármaco basado en nucleósidos con propiedades inhibidoras de la transcripción del ARN viral. Adicionalmente el LOPINAVIR, RITONAVIR y SOFOSBUVIR (17) parecen tener un efecto contra el síndrome respiratorio de Oriente Medio (MERS-
$\mathrm{CoV}$ ) en animales, pero desafortunadamente no en pacientes con COVID-19.

Recientemente, China ha aprobado el uso de FAVILAVIR, una droga antiviral usada para la influenza, como terapia alternativa no confirmada para COVID-19. A la fecha, cerca de 100 estudios clínicos están siendo realizados para encontrar nuevas terapias o repotenciar medicamentos usados para otras enfermedades virales (Figura 9). La CLOROQUINA (44) es un fármaco económico y usado en el tratamiento de la malaria, ha demostrado tener efecto positivo en pacientes chinos y franceses con la COVID-19 (45). Sin embargo, médicos americanos cuestionan dicho beneficio terapéutico. Otros estudios clínicos proponen la combinación de hidroxicloroquina/Azitromicina (46), pero dicho efecto es aún controversial (47). Otros estudios sugieren el uso de la inmunoterapia IgG, pero en estudios anteriores se ha observado que dicha estrategia causa la muerte rápida de monos de laboratorio.

Existen informes sobre un anticuerpo monoclonal (humano), el 47D11, que neutraliza al SARS-CoV-2, uniéndose a un epítopo conservado en el dominio de Unión al receptor de espiga, lo que explica su capacidad para neutralizar de forma cruzada a SARS-CoV y SARSCoV-2, utilizando un mecanismo que es independiente de la inhibición de la unión al receptor, ofreciendo así una posibilidad para la prevención y el tratamiento de COVID-19 (48).

Otros estudios mencionan que un objetivo farmacológico atractivo en el coronavirus es la proteasa principal (Mpro, 3CLpro) (49), debido a su rol esencial en el procesamiento de las poliproteínas que son traducidas del RNA viral; Linling Zhang et al. han desarrollado inhibidores mejorados de $\alpha$-cetoamida, que contienen piridona y actúan como potentes inhibidores del SARS-CoV-2 Mpro (50).

Una estrategia terapéutica podría encontrarse en el plasma de pacientes que superaron los síntomas del COVID-19. El procedimiento de extracción del plasma es conocido y puede llevarse a cabo en los laboratorios o hospitales. Esta estrategia podría ayudar al estado a controlar el brote de la transmisión mientras que la nueva vacuna contra el SARSCoV-2 esté disponible.

La formación de equipos clínicos multidisciplinarios es muy importante para el manejo de los pacientes con COVID-19, dado que el SARS-CoV-2 ataca a las células pulmonares (neumocitos) (51,52), células del intestino (53), corazón y vasos sanguíneos (54). En los pulmones el CoV-2 bloquea la formación de moléculas surfactantes (detergentes) las cuales limpian y mantienen las vías aéreas abiertas (52). Esto ocasiona dificultad para respirar y disminución dramática de los niveles de O2. Nuevas observaciones en China sugieren que el COVID-19 puede producir la formación de células sanguíneas anormales, ocasionado al paciente problemas a nivel del bazo y complicaciones sintomáticas, sobre todo en el grupo de alto riesgo (18).

Los virus COV1 y COV8 secuestran el hierro de la sangre causando la interrupción de la formación de la hemoglobina $(55,56)$. Normalmente, los niveles de azúcar en la sangre aumentan en personas mayores (57), lo que incrementa la cantidad de la hemoglobina glicosilada (HbA1c) $(58,59)$. Esto hace que el virus pueda interferir con el grupo hemo en los glóbulos rojos destruyendo los pulmones y reduciéndola capacidad de oxigenación. Esto nos indica que los pacientes 
se deben someter a una dieta alimenticia bien planificada, alta en fibras y proteína de origen vegetal y baja en azucares y carbohidratos. Además, mediciones de niveles anormales de hierro y HbA1c podrían asociarse a la contaminación del virus y usarse como parte del despistaje en pacientes asintomáticos. La predicción de tratamientos efectivos como las vacunas estarán disponibles para junio del 2021, es por eso que medidas alternativas deberán ser tomadas basadas en las experiencias de otros países como China e Italia. Los expertos sugieren que la toma de decisiones desde un punto teórico y/o académico podría ser peor que la solución. Por eso se recomienda seguir la evolución de otros países donde el brote se encuentra en descenso y aplicar las medidas tomadas.

\section{Detener y/o reducir la propagación del virus}

Las recomendaciones para detener y/o reducir la propagación del virus se basan en:

- Anticipar y reducir el uso de hospitales y personal médico por casos ajenos al brote.

- Priorizar la protección y bioseguridad del personal médico para evitar expansión de la transmisión hospitalaria.

- Proveer el cuidado médico correcto al paciente para maximizar el uso de instalaciones hospitalarias.

- Extender la capacidad de diagnóstico para maximizar el uso de instalaciones hospitalarias.

- Establecer protocolos de aislamiento para minimizar la transmisión del SARS-CoV-2.

Las pandemias crean demasiada incertidumbre, pero lo que es cierto es que el ingenio de las autoridades de la salud pública y colaboración con los sistemas hospitalarios pueden ser críticos e importantes para mejorar y establecer las estrategias que cumplan los requerimientos de esta enfermedad aun en evolución.

\section{Manejo de poblaciones, tráfico aéreo y turismo}

Actualmente se ha demostrado que el aislamiento social e inmovilización de personas, ciudades e incluso de países enteros, ayuda a detener el crecimiento de la curva del brote de la epidemia. Un claro ejemplo es lo ocurrido en China donde se puedo observar que el aislamiento de la población redujo el R0 o número de transmisiones. Esto dio más tiempo y recursos a los equipos médicos para tratar a los pacientes sin tener que ocuparse en casos nuevos. Sin embargo, la aplicación de cuarentena sin previo estudio socioeconómico puede ser dañinas para un país.

En el caso de Perú, 229736 casos han sido confirmados (junio 14, 2020). Esto ha originado que el gobierno cierre todas las fronteras aéreo, terrestre, marítimo y fluvial. Se decretó inamovilidad nacional desde el 16 marzo hasta el 30 de junio. En contraparte, estudios socioeconómicos y epidemiológicos sugieren que estas medidas deben estar acompañadas de una buena estrategia sanitaria de prevención, tratamiento y mitigación de la epidemia para obtener resultados óptimos. De otro modo solo se estaría retardando el brote de la epidemia en el país. Por eso es necesario establecer colaboraciones con médicos de países que hayan afrontado las etapas que están por venir en el Perú.
Dado que Lima cuenta con el $\sim 57 \%$ de casos confirmados, las últimas medidas del estado peruano serían las correctas. Sin embargo, otras ciudades con un número bajo de casos confirmados podrían tener cuarentenas intermitentes o reducir la concentración de personas, permitiendo así el desarrollo socioeconómico del país.

El control del tráfico aéreo es primordial ya sea doméstico o internacional. Se ha observado que los ciudadanos europeos y americanos tienden a movilizarse a ciudades o países con bajo índice de casos confirmados. Esto causaría que nuevos casos provengan de la migración de personas presuntamente sanas. Actualmente, el Perú ha cerrado el transporte terrestre y aéreo interprovincial, disminuyendo el riesgo de propagación del virus.

En vista de ello, y si se mantienen los datos, podemos sugerir tomar las medidas siguientes:

- Mantener las medidas excepcionales, debido a que con ello se lograría la contención adecuada de la propagación del proceso infeccioso.

- Evitar la movilización interprovincial de los pobladores de ciudades con alta densidad de casos hacia las ciudades con bajos casos confirmados.

- Incrementar exponencialmente la capacidad de diagnóstico descentralizado.

- Proveer equipamiento de bio-protección adecuado al personal médico, técnico y de limpieza de centros hospitalarios.

- Crear la geolocalización de zonas de casos positivos en forma digital y virtual.

- Campaña agresiva por medios de comunicación de mantener las medidas de protección pertinentes, uso de jabón en los lavados de manos en forma constante, limpieza de superficies con agua de jabón, con la máxima frecuencia posible.

- Consumo de alimentos que pudieran fortalecer su sistema inmunológico.

\section{Preparación para el invierno en el Peru y el segundo ciclo de la pandemia}

El brote del virus SARS-CoV-2 estuvo desapercibido por muchos países dado que esto ocurrió durante el comienzo del invierno en Asia, Europa y Norte América, donde habitualmente se incrementan los brotes de resfriados comunes. Lo que pudo haber sido un factor determinante para que el virus se disemine globalmente. A la fecha dichos continentes han salido de la estación fría, lo cual permitirá ver claramente los casos de COVID-19 sin la variable del efecto del clima en la población de esos países. En contra parte, el Perú está terminado la estación calurosa para empezar el invierno. Esto podría ser muy desastroso para la economía y el sistema sanitario, si el gobierno no continua con las acciones para mitigar el brote del virus antes de la llegada de la estación de invierno. Para esto se recomienda campañas de vacunación contra la influenza y el neumococo.

A parte del factor climatológico, el mundo debe prepararse para un segundo brote de la pandemia o como lo indican los especialistas el segundo ciclo del brote. Para esto el Perú deberá haber cubierto las deficiencias observadas actualmente con la implementación de laboratorios científicos trabajando en la búsqueda de tratamientos y con 
capacidades de diagnóstico por arriba de $>1000$ análisis por día. Cabe resaltar que las vacunas y nuevos medicamentos serán desarrollados en base a estudios en personas de origen europeo y asiático. Lo cual podría significar una deficiencia de efecto terapéutico en otras etnias como la peruana. El gobierno deberá motivar y financiar estudios genéticos y moleculares para determinar la respuesta de los pacientes a la terapia. Así mismo iniciar la fabricación de ventiladores y equipamiento necesario para que los pacientes puedan ser asistidos en casos severos.

\section{REFERENCIAS BIBLIOGRÁFICAS}

1. Hoffmann M, Kleine-Weber H, Schroeder S, Krüger N, Herrler T, Erichsen S, et al. SARS-CoV-2 cell entry depends on ACE2 and TMPRSS2 and is blocked by a clinically proven protease inhibitor. Cell. 2020.

2. Vankadari N, Wilce JA. Emerging COVID-19 coronavirus: glycan shield and structure prediction of spike glycoprotein and its interaction with human CD26. Emerging microbes \& infections. 2020;9(1):601-4.

3. Wang C, Horby PW, Hayden FG, Gao GF. A novel coronavirus outbreak of global health concern. The Lancet. 2020;395(10223):470-3.

4. Liu W, Zhang Q, Chen J, Xiang R, Song H, Shu S, et al. Detection of Covid-19 in children in early January 2020 in Wuhan, China. New England Journal of Medicine. 2020;382(14):1370-1.

5. Van Doremalen N, Bushmaker T, Morris DH, Holbrook MG, Gamble A, Williamson BN, et al. Aerosol and surface stability of SARS-CoV-2 as compared with SARS-CoV-1. New England Journal of Medicine. 2020;382(16):1564-7.

6. Ong SWX, Tan YK, Chia PY, Lee TH, Ng OT, Wong MSY, et al. Air, surface environmental, and personal protective equipment contamination by severe acute respiratory syndrome coronavirus 2 (SARS-CoV-2) from a symptomatic patient. Jama. 2020;323(16):16102.

7. Liu Y, Ning Z, Chen Y, Guo M, Liu Y, Gali NK, et al. Aerodynamic analysis of SARS-CoV-2 in two Wuhan hospitals. Nature. 2020:1-4.

8. Kampf G, Todt D, Pfaender S, Steinmann E. Persistence of coronaviruses on inanimate surfaces and their inactivation with biocidal agents. Journal of Hospital Infection. 2020;104(3):246-51.

9. Wang D, Hu B, Hu C, Zhu F, Liu X, Zhang J, et al. Clinical characteristics of 138 hospitalized patients with 2019 novel coronavirus-infected pneumonia in Wuhan, China. Jama. 2020;323(11):1061-9.

10. Kakimoto K, Kamiya H, Yamagishi T, Matsui T, Suzuki $\mathrm{M}$, Wakita T. Initial investigation of transmission of COVID-19 among crew members during quarantine of a cruise ship_Yokohama, Japan, February 2020. 2020.

11. Egloff C, Vauloup-Fellous C, Picone O, Mandelbrot L, Roques P. Evidence and possible mechanisms of rare maternal-fetal transmission of SARS-CoV-2. Journal of Clinical Virology. 2020:104447.

12. Zhang J-j, Dong X, Cao Y-y, Yuan Y-d, Yang Y-b, Yan Y-q, et al. Clinical characteristics of 140 patients infected with SARS-CoV-2 in Wuhan, China. Allergy. 2020.

13. Huang C, Wang Y, Li X, Ren L, Zhao J, Hu Y, et al. Clinical features of patients infected with 2019 novel coronavirus in Wuhan, China. The lancet. 2020;395(10223):497-506.

14. Del Rio C, Malani PN. COVID-19 - new insights on a rapidly changing epidemic. Jama. 2020;323(14):133940.

15. Li T. Diagnosis and clinical management of severe acute respiratory syndrome coronavirus 2 (SARSCoV-2) infection: an operational recommendation of Peking Union Medical College Hospital (V2. 0) working group of 2019 novel coronavirus, Peking union medical college hospital. Emerging microbes \& infections. 2020;9(1):582-5.

16. Ashour HM, Elkhatib WF, Rahman M, Elshabrawy HA. Insights into the recent 2019 novel coronavirus (SARSCoV-2) in light of past human coronavirus outbreaks. Pathogens. 2020;9(3):186.

17. Tetro JA. Is COVID-19 receiving ADE from other coronaviruses? Microbes and infection. 2020;22(2):723.

18. Han H, Yang L, Liu R, Liu F, Wu K-1, Li J, et al. Prominent changes in blood coagulation of patients with SARS-CoV-2 infection. Clinical Chemistry and Laboratory Medicine (CCLM). 2020;1(ahead-of-print).

19. Zhang X, Cai H, Hu J, Lian J, Gu J, Zhang S, et al. Epidemiological, clinical characteristics of cases of SARS-CoV-2 infection with abnormal imaging findings. International Journal of Infectious Diseases. 2020.

20. Xu X-W, Wu X-X, Jiang X-G, Xu K-J, Ying L-J, Ma $\mathrm{C}-\mathrm{L}$, et al. Clinical findings in a group of patients infected with the 2019 novel coronavirus (SARSCov-2) outside of Wuhan, China: retrospective case series. bmj. 2020;368.

21. Young BE, Ong SWX, Kalimuddin S, Low JG, Tan SY, Loh J, et al. Epidemiologic features and clinical course of patients infected with SARS-CoV-2 in Singapore. Jama. 2020;323(15):1488-94.

22. Liu J, Zheng X, Tong Q, Li W, Wang B, Sutter K, et al. Overlapping and discrete aspects of the pathology and pathogenesis of the emerging human pathogenic coronaviruses SARS-CoV, MERS-CoV, and 2019nCoV. Journal of medical virology. 2020;92(5):491-4.

23. Liu H, Liu F, Li J, Zhang T, Wang D, Lan W. Clinical and CT imaging features of the COVID-19 pneumonia: Focus on pregnant women and children. Journal of infection. 2020.

24. Ralph R, Lew J, Zeng T, Francis M, Xue B, Roux M, et al. 2019-nCoV (Wuhan virus), a novel Coronavirus: human-to-human transmission, travel-related cases, and vaccine readiness. The Journal of Infection in Developing Countries. 2020;14(01):3-17.

25. Lueers J-C, Klußmann JP, Guntinas-Lichius O. The Covid-19 pandemic and otolaryngology: What it comes down to? Laryngo-rhino-otologie. 2020.

26. Porcheddu R, Serra C, Kelvin D, Kelvin N, Rubino S. Similarity in case fatality rates (CFR) of COVID-19/ SARS-COV-2 in Italy and China. The Journal of Infection in Developing Countries. 2020;14(02):125-8. 
27. Yang S, Cao P, Du P, Wu Z, Zhuang Z, Yang L, et al. Early estimation of the case fatality rate of COVID-19 in mainland China: a data-driven analysis. Annals of translational medicine. 2020;8(4).

28. Shi S, Qin M, Shen B, Cai Y, Liu T, Yang F, et al. Association of cardiac injury with mortality in hospitalized patients with COVID-19 in Wuhan, China. JAMA cardiology. 2020.

29. Mizumoto K, Chowell G. Estimating Risk for Death from 2019 Novel Coronavirus Disease, China, January-February 2020. Retrieved from Volume 26, Number 6-June 2020. Center for Disease Control and Prevention-CDC https://doi org/103201/eid2606. 2020;200233.

30. Zhou F, Yu T, Du R, Fan G, Liu Y, Liu Z, et al. Clinical course and risk factors for mortality of adult inpatients with COVID-19 in Wuhan, China: a retrospective cohort study. The lancet. 2020.

31. Chen Y, Peng J. Treatment strategy for gastrointestinal tumor under the outbreak of novel coronavirus pneumonia in China. Zhonghua wei chang wai ke za zhi= Chinese journal of gastrointestinal surgery. 2020;23(2):I-IV.

32. Park WB, Kwon N-J, Choi S-J, Kang CK, Choe PG, Kim JY, et al. Virus isolation from the first patient with SARS-CoV-2 in Korea. Journal of Korean Medical Science. 2019;35(7).

33. Emma HB. Preliminary case report on the SARSCoV-2 cluster in the UK, France, and Spain. SWISS MEDICAL WEEKLY. 2020;150.

34. Wang W, Xu Y, Gao R, Lu R, Han K, Wu G, et al. Detection of SARS-CoV-2 in different types of clinical specimens. Jama. 2020;323(18):1843-4.

35. Pfefferle S, Reucher S, Nörz D, Lütgehetmann $M$. Evaluation of a quantitative RT-PCR assay for the detection of the emerging coronavirus SARS-CoV-2 using a high throughput system. Eurosurveillance. 2020;25(9):2000152.

36. Liu R, Han H, Liu F, Lv Z, Wu K, Liu Y, et al. Positive rate of RT-PCR detection of SARS-CoV-2 infection in 4880 cases from one hospital in Wuhan, China, from Jan to Feb 2020. Clinica Chimica Acta. 2020.

37. Elfiky AA. Anti-HCV, nucleotide inhibitors, repurposing against COVID-19. Life sciences. 2020:117477.

38. Zhang T, Cui X, Zhao X, Wang J, Zheng J, Zheng G, et al. Detectable SARS-CoV-2 viral RNA in feces of three children during recovery period of COVID-19 pneumonia. Journal of Medical Virology. 2020.

39. Organization WH. Laboratory biosafety guidance related to the novel coronavirus $(2019-\mathrm{nCoV})$. Interim guidance Available from URL: https://www who int/ docs/defaultsource/coronaviruse/laboratory-biosafetynovel-coronavirus-version-1-1 pdf. 2020.

40. Li H, Zhou Y, Zhang M, Wang H, Zhao Q, Liu J. Updated approaches against SARS-CoV-2. Antimicrobial agents and chemotherapy. 2020;64(6).

41. Martinez MA. Compounds with therapeutic potential against novel respiratory 2019 coronavirus. Antimicrobial agents and chemotherapy. 2020;64(5).

42. Zhang C, Huang S, Zheng F, Dai Y. Controversial treatments: an updated understanding of the Coronavirus Disease 2019. Journal of medical virology. 2020.
43. Lai C-C, Shih T-P, Ko W-C, Tang H-J, Hsueh P-R. Severe acute respiratory syndrome coronavirus 2 (SARSCoV-2) and corona virus disease-2019 (COVID-19): the epidemic and the challenges. International journal of antimicrobial agents. 2020:105924.

44. Yao X, Ye F, Zhang M, Cui C, Huang B, Niu P, et al. In vitro antiviral activity and projection of optimized dosing design of hydroxychloroquine for the treatment of severe acute respiratory syndrome coronavirus 2 (SARS-CoV-2). Clinical Infectious Diseases. 2020.

45. Devaux CA, Rolain J-M, Colson P, Raoult D. New insights on the antiviral effects of chloroquine against coronavirus: what to expect for COVID-19? International journal of antimicrobial agents. 2020:105938.

46. Gautret P, Lagier J-C, Parola P, Meddeb L, Mailhe M, Doudier B, et al. Hydroxychloroquine and azithromycin as a treatment of COVID-19: results of an open-label non-randomized clinical trial. International journal of antimicrobial agents. 2020:105949.

47. Wang M, Cao R, Zhang L, Yang X, Liu J, Xu M, et al. Remdesivir and chloroquine effectively inhibit the recently emerged novel coronavirus (2019-nCoV) in vitro. Cell research. 2020;30(3):269-71.

48. Kruse RL. Therapeutic strategies in an outbreak scenario to treat the novel coronavirus originating in Wuhan, China. F1000Research. 2020;9.

49. Liu W, Morse JS, Lalonde T, Xu S. Learning from the past: possible urgent prevention and treatment options for severe acute respiratory infections caused by 2019 nCoV. Chembiochem. 2020.

50. Zhang L, Lin D, Sun X, Curth U, Drosten C, Sauerhering L, et al. Crystal structure of SARS-CoV-2 main protease provides a basis for design of improved $\alpha$-ketoamide inhibitors. Science. 2020;368(6489):40912.

51. Tian S, Hu W, Niu L, Liu H, Xu H, Xiao S-Y. Pulmonary pathology of early phase 2019 novel coronavirus (COVID-19) pneumonia in two patients with lung cancer. Journal of Thoracic Oncology. 2020.

52. Fu Y, Cheng Y, Wu Y. Understanding SARS-CoV-2mediated inflammatory responses: from mechanisms to potential therapeutic tools. Virologica Sinica. 2020:1-6.

53. de Patología Digestiva SE. Asociación Española de Gastroenterología. Recommendations by the SEPD and AEG, both in general and on the operation of gastrointestinal endoscopy and gastroenterology units, concerning the current SARS-CoV-2 pandemic (March, 18). Rev Esp Enferm Dig. 2020;3:23.

54. Madjid M, Safavi-Naeini P, Solomon SD, Vardeny O. Potential effects of coronaviruses on the cardiovascular system: a review. JAMA cardiology. 2020.

55. Henry BM. COVID-19, ECMO, and lymphopenia: a word of caution. The Lancet Respiratory Medicine. 2020;8(4):e24.

56. Chan KW, Wong VT, Tang SCW. COVID-19: An update on the epidemiological, clinical, preventive and therapeutic evidence and guidelines of integrative Chinese-Western medicine for the management of 2019 novel coronavirus disease. The American journal of Chinese medicine. 2020;48(03):737-62.

57. Al-Sofiani ME, Ganji S, Kalyani RR. Body composition 
changes in diabetes and aging. Journal of Diabetes and its Complications. 2019.

58. Preshaw PM, Bissett SM. Periodontitis and diabetes. BDJ Team. 2020;7(5):27-35.

59. Xu K, Cai H, Shen Y, Ni Q, Chen Y, Hu S, et al. Management of corona virus disease-19 (COVID-19): the Zhejiang experience. Journal of Zhejiang University (medical science). 2020;49(1):0.

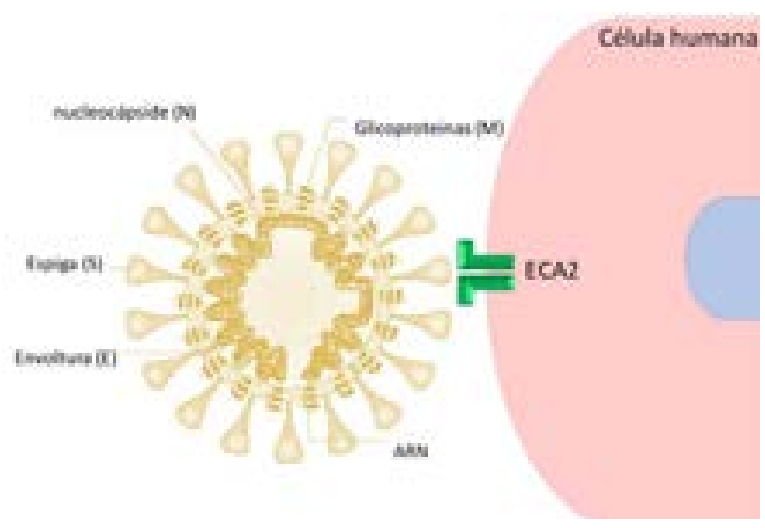

Fig. 1: El virus SARS-CoV-2 interacciona con el receptor ECA2 para ingresar a las células humanas.

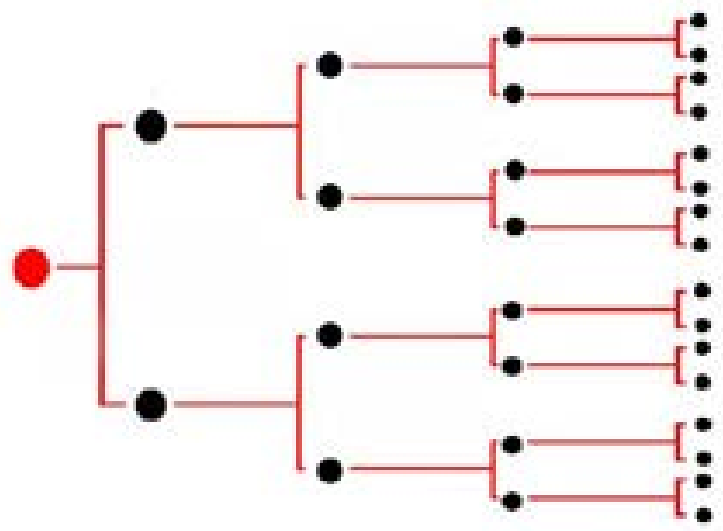

Fig. 2: R0 explica el número de casos nuevos que una persona infectada puede causar. El ejemplo en esta figura refiere a un $\mathrm{R}=\mathbf{2}$

Tabla 1: Numero de Contagiados hasta el día de 01/04/2020

\begin{tabular}{cccccccccccccccc}
\hline Tiempo & 1 & 2 & 3 & 4 & 5 & 6 & 7 & 8 & 9 & 10 & 11 & 12 & 13 & 14 & 15 \\
\hline Contagiados & 1 & 1 & 6 & 8 & 11 & 11 & 15 & 28 & 38 & 43 & 86 & 117 & 145 & 234 & 234 \\
\hline Tiempo & 16 & 17 & 18 & 19 & 20 & 21 & 22 & 23 & 24 & 25 & 26 & 27 & & & \\
\hline Contagiados & 318 & 363 & 395 & 416 & 480 & 580 & 635 & 671 & 852 & 950 & 1065 & 1323 & &
\end{tabular}

a)Los tiempos son en días; b)Los contagiados en unidades de pacientes que dieron positivo a la prueba de corona virus.

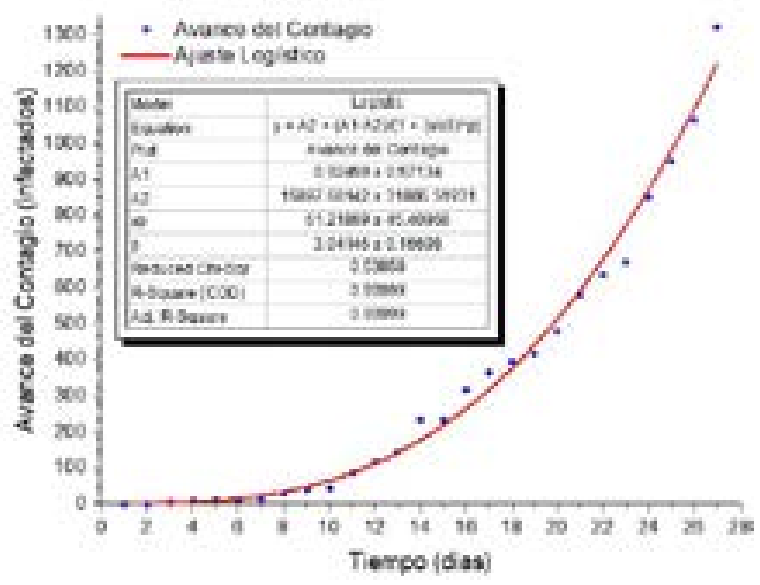

Fig. 3: Grafica de datos análisis no-lineal

$$
f(x)=A 2+\frac{(A 1-A 2)}{1+\left(\frac{x}{x 0}\right)^{P}} \quad f^{\prime}(x)=-\frac{(A 1-A 2) P\left(\frac{x}{m}\right)^{p-1}}{m\left(1+\left(\frac{x}{m}\right)^{p}\right)^{2}}
$$

Fórmula 1. Ecuación matemática y su tasa de variación

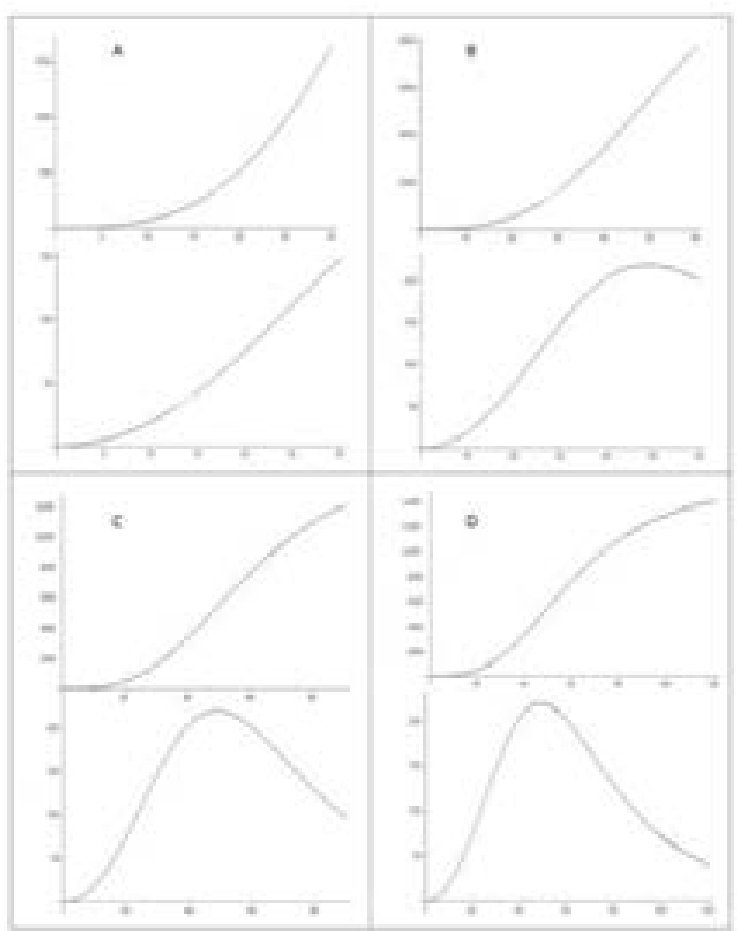

Fig 4: Grafica de la ecuación modelo y tasa de variación para el caso peruano a los 30 (A), 60 (B), 90 (C) y 120 días (D) 
Tabla 2: Clasificación de síntomas

\begin{tabular}{|c|c|c|c|}
\hline Síntomas & COVID-19 & $\begin{array}{l}\text { Resfriado } \\
\text { común }\end{array}$ & Gripe por influenza \\
\hline Fiebre & Frecuente & Poco frecuente & Frecuente \\
\hline Tos & Frecuente, seca & Moderada & Moderada, seca \\
\hline Cansancio & Frecuente & Poco frecuente & Frecuente \\
\hline $\begin{array}{c}\text { Dificultad } \\
\text { respiratoria }\end{array}$ & Frecuente & Ninguna & Ninguna \\
\hline Dolor de Cabeza & Poco frecuente & Raramente & Frecuente \\
\hline Dolor de garganta & Poco frecuente & Frecuente & Poco frecuente \\
\hline Escalofríos & Poco frecuente & Poco frecuente & Frecuente \\
\hline Mucosidad nasal & Raramente & Frecuente & Poco frecuente \\
\hline Estornudo & Raramente & Frecuente & Poco frecuente \\
\hline Diarrea & Poco frecuente & Raramente & Poco frecuente \\
\hline
\end{tabular}

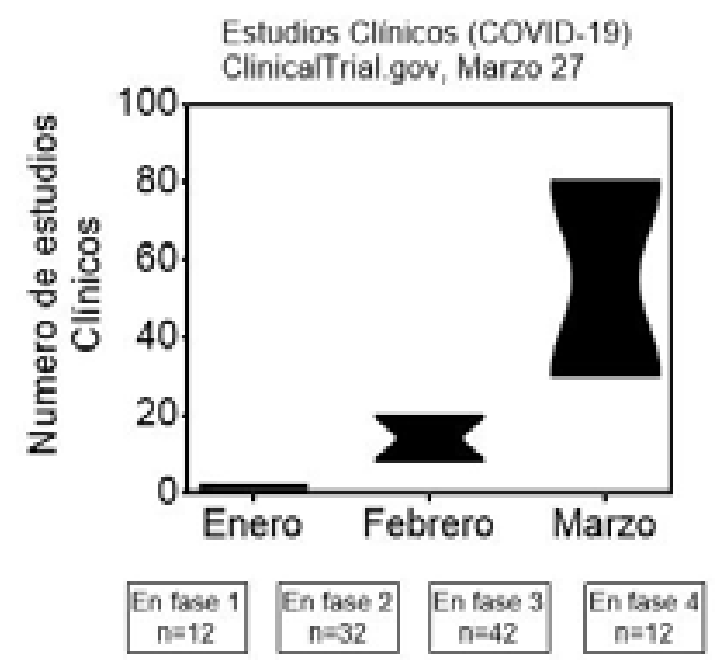

Fig. 5: Estudios clínicos enfocados en la inhibición del virus SARS-CoV-2 o complicaciones Clínicas en pacientes con la COVID-19 